\title{
Effects of pH, ions, and thermal treatments on physical stability of astaxanthin nanodispersions
}

\begin{abstract}
In this work, astaxanthin nanodispersions were prepared using selected three component stabilizer system through a solvent-diffusion technique, with the particle size of $98.3 \mathrm{~nm}$. The stability of produced nanodispersions against $\mathrm{pH}$, salts, and heating were then evaluated. The produced nanodispersions exhibited good physical stability under wide ranges of $\mathrm{pH}$ (except around isoelectric point), sodium ion concentrations, and relatively high-temperature treatments (up to $60^{\circ} \mathrm{C}$ ). However, formation of large particles was observed in either presence of calcium ions or higher thermal treatments (more than $60^{\circ} \mathrm{C}$ ).
\end{abstract}

Keyword: Astaxanthin; Nanodispersions; Physical stability 